\title{
Evaluación de la Felicidad: Análisis Psicométrico de la Escala de Felicidad Subjetiva en Población Chilena
}

\author{
Evaluation of Happiness: Psychometric Analysis of the Subjective Happiness Scale in \\ Chilean population
}

\author{
Pablo Vera-Villarroel \\ Karem Celis-Atenas \\ $\mathrm{y}$ \\ Natalia Córdova-Rubio \\ Universidad de Santiago de Chile, USACH, Chile \\ (Recibido: 15 de abril de 2010; Acep: 24 de noviembre de 2010)
}

\begin{abstract}
Resumen
El objetivo de esta investigación fue evaluar las propiedades psicométricas de la escala de Felicidad Subjetiva (Lyubomirsky \& Lepper, 1999). El objetivo del primer estudio, en que participaron 300 personas de población general, fue estimar confiabilidad (consistencia interna y estabilidad temporal) y validez de constructo de la escala. El segundo estudio, donde participaron 779 personas, tuvo por objetivo obtener indicadores definitivos de confiabilidad y validez en muestras con rangos etarios diversos. Además, evaluó validez convergente y divergente con los instrumentos: Inventario de Depresión de Beck (BDI), Cuestionario de los Cinco Grandes (BFI), y Cuestionario de Optimismo Disposicional (LOT-R). Los principales resultados del primer estudio muestran un alfa de .78 y estabilidad temporal de .61 ( 8 semanas de diferencias) y una estructura factorialmente pura. Para el segundo estudio la estimación de la confiabilidad estuvo entre .73 y .87 en las diversas muestra estudiadas; se encontró una estructura factorialmente pura; las correlaciones entre la escala de felicidad subjetiva y los instrumentos utilizados para evaluar validez convergente y divergente fueron adecuadas y altamente significativas en la línea de lo esperado teóricamente. La evidencia indica adecuación de la escala para su utilización en la evaluación de este constructo en población chilena.
\end{abstract}

Palabras clave: Psicología positiva, felicidad, análisis psicométrico.

\begin{abstract}
The purpose of this study was to evaluate the psychometric properties of the subjective happiness scale (Lyubomirsky \& Lepper, 1999). The objective of the first study involving 300 people from the general population was the estimated reliability (internal consistency and temporal stability) and construct validity of the scale. The second study involved 779 people, its purpose was to obtain definitive indicators of reliability and validity in samples with diverse age range. In addition, convergent and divergent validity was evaluated with instruments: Beck Depression Inventory (BDI), Big Five Questionnaire (BFI), and Dispositional Optimism Questionnaire (LOT-R). The main results of study showed an alpha of .78 and temporal stability of 61 (8 weeks apart) and a pure factorial structure. For the second study, the reliability estimation was between .73 and .87 according to the many samples studied, was found a pure factorial structure, the correlations between subjective happiness scale and instruments used to assess convergent and divergent validity were adequate, highly significant and in line with theoretical expectations. The evidence indicates adequacy of the scale for use in the assessment of this construct in the Chilean population.
\end{abstract}

Key words: Positive psychology, happiness, properties psychometric 


\section{Introducción}

Los seres humanos tenemos como meta común alcanzar bienestar y felicidad (Buss, 2000). En las últimas décadas se ha atestiguado una explosión en la investigación sobre la felicidad o bienestar subjetivo (Lyubomirsky \& Lepper, 1999; Urzúa, Cortés, Vega, Prieto, Tapia \& 2009; ZegersPrado, Rojas-Barahona, Förster-Marín, 2009) y actualmente este interés se extiende desde las áreas de gestión hasta la salud mental (Steel, Schmidt \& Shultz, 2008).

Incluso en disciplinas fuera de la psicología como la economía, emergen nuevas áreas con el objetivo de examinar los determinantes socioeconómicos y macropolíticos de la felicidad (Blanchflower \& Oswald, 2004; Blanchflower \& Oswald, 2005; Di Tella, MacCulloch \& Oswald, 2003; Easterlin, 2001); tornando estos constructos en indicadores relevantes para ser considerados en políticas públicas (Cornelis, 2010).

La investigación en bienestar parece ser especialmente prominente en la psicología empírica (para una revisión Ryan \& Deci, 2001). Los estudios muestran su relación con diversos constructos como por ejemplo, parámetro fisiológicos e inmunitarios (Barak, 2006) y constructos relacionados (Cuadra-Peralta, Veloso-Besio, IbergarayPérez \& Rocha-Zúñiga, 2010; Retana-Franco \& SánchezAragón. 2010).

Las personas felices tienen éxito a través de múltiples dominios de la vida, incluyendo el matrimonio, la amistad, los ingresos, el desempeño del trabajo y la salud (Lyubomirsky, King \& Diener, 2005). Quienes se perciben a sí mismo como felices responden de manera más adaptativa a experiencias cotidianas, en la toma decisiones, en la percepción e interpretación de situaciones sociales y en la recuperación frente eventos negativos como el fracaso (Abbe, Tkach \& Lyubomirsky, 2003; Lyubomirsky, et al., 2005; Lyubomirsky, Sheldon, \& Schkade, 2005; Lyubomirsky $\&$ Tucker, 1998). Así, la relación entre éxito y felicidad existe y ocurre no sólo porque el éxito hace la felicidad, sino que también porque el afecto positivo engendra éxito (Lyubomirsky, et al., 2005).

La evidencia empírica ha motivado en psicología clínica el desarrollo de intervenciones que muestran que los niveles de felicidad son susceptibles de ser incrementados (Abbe, et al., 2003; Green, Gardner \& Reid, 1997; Lyubomirsky, et al., 2005; Otake, Shimai, Tanaka, Otsui \& Frederickson 2006; Seligman, Steen, Park \& Peterson, 2005).

Este exponencial aumento de estudios e intervenciones, demandan el perfeccionamiento de instrumentos de evaluación válidos y estandarizados. No obstante, existen desacuerdos en la validez, confiabilidad y comparabilidad de las medidas de felicidad y bienestar (Cornelis, 2010; para una revisión Johns \& Ormerod, 2007; Lim, 2008).
Algunos autores plantean que se ha descuidado la tarea de definir las características esenciales del bienestar psicológico (Ryff, 1989) lo que se refleja en la diversidad de conceptos asociados a felicidad, bienestar subjetivo, calidad de vida, afectividad positiva los cuales frecuentemente se utilizan como términos intercambiables.

De esta forma, nos encontramos con diversas escalas para evaluar bienestar que se asocian a la evaluación de la felicidad a nivel teórico y en su contenido temático o en sus dimensiones. Así tenemos entre otros, el Satisfaction with life scale (Pavot \& Diener, 1999), The Short DepressionHappiness Scale (SDHS) (Joseph, Linley, Harwood, Lewis \& McCollam, 2004), Oxford Happinnes Inventory (Lewis, Francis \& Zieberts, 2002), the Steen Happiness Index (SHI) (Seligman, et al., 2005), escala factorial de felicidad, (Alarcón, 2006), que intercambian los conceptos antes señalados. En general estas escalas no evalúan un constructo unitario y se identifican componentes compuestos por las dimensiones afecto positivo, afecto negativo y un componente cognitivo satisfacción vital (Pavot \& Diener, 1993; Vera-Villarroel et al., 2010). Estos instrumentos multi-item han sido criticadas por evaluar diferentes componentes de la felicidad (Swami, 2008) y lo difuso del dominio temático de los ítems, por lo que se ha apelado al uso de enfoques conceptuales más estrictos para evitar el solapamiento con otros constructos (Kashdan, 2004). Por otra parte, las escalas de un único ítem que realizan una evaluación global no son conducente para evaluar sus propiedades psicométricas (Swami, 2008).

Como respuesta a estas dificultades, a nivel internacional se destaca la "Subjective Happiness Scale" o Escala de Felicidad Subjetiva, desarrollada por Sonja Lyubomirsky \& Heidi Lepper (1999). Ésta escala va más allá de la suma de estados emocionales positivos y negativos, y cogniciones relacionadas con el fenómeno, es una medida general de felicidad subjetiva, evaluando una categoría molar de bienestar como fenómeno psicológico global (Lyubomirsky \& Lepper, 1999).

La Escala de Felicidad Subjetiva considera la felicidad desde la perspectiva de quien responde, asumiendo el supuesto que aún cuando existen diversos motivos para ser feliz, la mayoría de las personas tiene su propia idea de qué es ser feliz, cuando lo son o cuando no lo son, siendo capaces de reportarlo (Lyubomirsky, 2008).

Es una medida de felicidad de 4 items, validada inicialmente en muestras estadounidenses y rusas (Lyubomirsky \& Lepper, 1999). Entre otras se cuenta con la versión China, Malaya, Germana y Filipina, las que replican la estructura unidimensional, muestran una buena consistencia interna y buena validez convergente con otras medidas de bienestar subjetivo (Swami, 2008; Swami, Voracek, Dressler, Eisma \& Furnham, 2009). 
La comparación de niveles de felicidad entre grupos y realizada con otras escalas muestra que existen diferencias culturales, etáreas y de género en la evaluación de la felicidad (Alesina, Di Tella, \& MacCulloch, 2004; Csikszentmihalyi, \& Hunter, 2003; Inglehart, Foa, Peterson \& Welzel, 2008; Mroczek \& Kolarz, 1998; Swami, et al., 2009; Yang, 2008). Es de presumir que las construcciones teóricas acerca de la felicidad y los instrumentos que la miden puedan encontrarse afectados por factores culturales de los países de origen (Alarcon, 2006). Por tanto, se deben realizar los análisis psicométrico pertinentes para su validación a otro contexto cultural, con muestras amplias en rangos etarios. Así, el objetivo de este estudio es analizar las propiedades psicométricas de la escala de felicidad subjetiva en población adolescente, universitarios y población general chilena.

\section{Método}

\section{Estudio 1 .}

Objetivo: la finalidad de este estudio fue obtener indicadores preliminares de confiabilidad (consistencia interna y estabilidad temporal) y evaluar la estructura factorial (validez de constructo) de la escala de Felicidad Subjetiva en una muestra de población general de Santiago de Chile.

\section{Participantes}

La muestra estuvo compuesta por 300 personas de la ciudad de Santiago de Chile (119 hombres y 181 mujeres) entre 13 y 75 años, con una media de 25.41 (D.E. = 13.40).

\section{Instrumentos}

Escala de Felicidad Subjetiva (Lyubomirsky \& Lepper, 1999): es una medida global de felicidad subjetiva, que evalúa una categoría molar de bienestar como fenómeno psicológico global, considerando la definición de felicidad desde la perspectiva de quien responde (Lyubomirsky \& Lepper, 1999). Consta de 4 ítems con respuesta tipo lickert, su corrección se hace mediante la sumatoria de los puntajes obtenidos y se dividen en el número total de ítem (Lyubomirsky \& Lepper, 1999).

En cuanto al contenido temático dos ítems piden a quienes la responden, caracterizarse a sí mismos usando intervalos absolutos e intervalos relativos a los pares, mientras los otros dos ítems ofrecen una breve descripción sobre individuos felices e infelices y piden a los encuestados respondan hasta qué punto cada caracterización los describe (Lyubomirsky \& Lepper, 1999)

\section{Resultados}

La puntuación media en la Escala de Felicidad Subjetiva fue de 5.04 (D.E.=1.21), no se encontraron diferencias significativas entre hombres y mujeres $(\mathrm{t}=.689 ; \mathrm{p}=.491)$, la correlación ítems total estuvo entre 0.37 y 0.70 , todas altamente significativas. La estimación de la confiabilidad a través del Alpha de Cronbach, se obtuvo un valor de 0.78, la evaluación de la estabilidad temporal (con 8 semanas de diferencias) se obtuvo una correlación entre las mediciones de .61 ( $<<0.01)$. Los detalles de la descripción de los ítems ver tabla 1 .

Tabla 1:

Media, Desviación y Correlación Ítem Total, del cuestionario de Felicidad

\begin{tabular}{|c|c|c|c|}
\hline & Media & D.E. & \\
\hline 1. En general, me considero & 5.32 & 1.47 & $.70^{* *}$ \\
\hline 2. En comparación con la mayoría de mis iguales, me considero & 5.20 & 1.54 & $.69 * *$ \\
\hline $\begin{array}{l}\text { 3. Algunas personas son muy felices en general. Disfrutan de la vida independientemente } \\
\text { de lo que suceda, sacan el máximo provecho de todo. ¿Hasta qué punto lo describe esta } \\
\text { caracterización? }\end{array}$ & 4.99 & 1.55 & $.60 * *$ \\
\hline $\begin{array}{l}\text { 4. Por término general, algunas personas no son muy felices. Aunque no se encuentran } \\
\text { deprimidas, nunca parecen estar tan felices como podrían. ¿Hasta qué punto lo describe } \\
\text { esta caracterización? }\end{array}$ & 4.69 & 1.70 & $.37 * *$ \\
\hline Total & 5.04 & 1.70 & \\
\hline
\end{tabular}

** $\mathrm{p}<0.01$

El estadístico de Kaiser-Meyer-Olkin $(\mathrm{KMO}=.75)$ y Prueba de Esfericidad de Bartlett (397.24; $<<.01$ ), muestran el ajuste de la matriz de correlaciones para llevar a cabo el análisis factorial. La estructura factorial, tras la realización del análisis por componente principales y rotación varimax, muestra una solución de un solo factor que explica un $61.65 \%$ de la varianza, presentando los ítems una saturación a este factor entre 0.56 y 0.87 . 


\section{Estudio 2.}

Objetivo: Obtener los indicadores psicométricos definitivos de confiabilidad y validez de la escala de felicidad subjetiva en diversas muestras (adolescentes, universitarios y población general).

\section{Participantes:}

En el segundo estudio participaron 779 personas. De ellos, 170 adolescentes (77 hombres y 92 mujeres), con una edad media de 15.98 años (D.E. $=1.31$ ), con edad que fluctuaron entre 13 y 19 años; 92 estudiantes universitarios (30 hombres y 61 mujeres), con edades entre 18 y 31 años, con una media de 21 años (D.E. $=2.10)$ y 517 Adultos $(316$ hombres y 201 mujeres), con edades entre 18 y 77 años, con una media de 39.48 años (D.E. $=12.64)$. La comparación entre las muestras estudiadas (ANOVA), muestra que existen diferencias significativas entre los grupos etarios ( $\mathrm{p}<.01$ ), los análisis Post Hoc (Prueba Scheffe) muestran que las diferencias se encuentran entre las muestra de adultos y adolescentes $(\mathrm{p}<.01)$ y entre adultos y universitarios $(\mathrm{p}<.01)$, no existen diferencias entre adolescentes y universitarios $(\mathrm{p}=.42)$. Obteniendo una media los adultos de 5.33 (D.E. $=1.11$ ), Adolescentes de 4.90 (D.E. 1.32) y los estudiantes universitarios de 5.1 (D.E. $=1.12$ )

\section{Instrumentos}

Optimismo Disposicional. Test de Orientación Vital revisado (LOT-R; Scheier, Carver, \& Bridges, 1994). El LOT-R consta de 6 ítems (más 4 ítems de relleno para hacer menos evidente el contenido) en escala Likert de 5 puntos (Chico, 2002). De ellos, 3 están redactados en sentido positivo (dirección optimismo) y 3 en sentido negativo (dirección pesimismo), los que se revierten para obtener una puntuación total orientada hacia el polo de optimismo (Ferrando, Chico, \& Tous, 2002). En la adaptación al castellano original el alfa de Cronbach reportado fue de 0.78 y la correlación con el LOT original fue de 0.95 (Otero, Luengo, Romero, Gómez, \& Castro, 1998). La escala tiene una estructura clara con dos dimensiones parcialmente independientes; todos los ítems tienen un aceptable poder discriminativo y, en conjunto, ambas subescalas miden con razonable precisión en un rango bastante amplio de niveles del rasgo (Ferrando, et al., 2002). Las propiedades psicométricas en población chilena muestran un alpha de Cronbach de .65 y la estructura factorial muestra claramente dos factores asociados al optimismo y al pesimismo (Vera-Villarroel, Córdova-Rubio \& Celis-Atenas, 2009).

Inventario de Depresión de Beck, (BDI, revised version) (Beck, Rush, Shaw, \& Emery, 1983). Este cuestionario cuantifíca la severidad de síntomas cognitivos, afectivos, conductuales y psicofisiológicos de la depresión, no proporcionar un diagnóstico. El rango de la puntuación obtenida es de 0 a 63 puntos.

Las propiedades psicométricas de esta escala han sido evaluadas tanto en población clínica, como normal (Beck,
Steer \& Garbin, 1988; Ritterband \& Spielberger, 1996). En la validación española del cuestionario se reportan altos índices de consistencia interna ( 0.83 y 0.90$)$, los valores para el test retest oscilan entre 0.60 y 0.72 y los índices de validez convergente están entre 0.68 y 0.89 (Sanz \& Vázquez, 1998; Vázquez \& Sanz, 1997, 1999; Olmedilla-Zafra \& Ortega-Toro, 2009). Inventario de los 5 Grandes (Caprara, Barabarinelli, \& Borgogni, 1993, BFI), El objetivo del test es la descripción y evaluación de la personalidad a través de 5 factores: Extraversión o Energía (Extreversion); Amabilidad o Afabilidad (Agreeableness); Responsabilidad o Tesón (Conscientiousness); Neuroticismo o Estabilidad Emocional (Neuroticism) y Apertura a la Experiencia o Apertura Mental (Openess); En su versión en español consta de 44 ítems que evalúan las 5 dimensiones del modelo de los 5 grandes (John \& Srivastava, 1999).

La dimensión extraversión apunta a actividad y energía, sociabilidad y emociones positivas, la dimensión amabilidad apunta a orientación prosocial, altruismo, modestia, la dimensión responsabilidad, evalúa conducta dirigida a la meda, autocontrol, la dimensión neuroticismo, incluye tristeza, ansiedad, nerviosismo, tensión y finalmente la dimensión apertura a la experiencia evalúa la profundidad, apertura mental y las experiencia de vida (Benet-Martínez \& John, 1998).

En las instrucciones se solicita a la persona que responda respecto al grado de acuerdo o desacuerdo, en una escala likert de 5 puntos ( 1 Totalmente en desacuerdo a 5 Totalmente de acuerdo) respecto a diversas características propuestas. Para su corrección, el BFI, consta de ítems positivos y negativos; por lo que es necesario invertir los negativos, para luego promediar el resultado (John \& Srivastava, 1999).

Respecto a sus propiedades psicométricas en muestra española, se obtuvo alfas entre .84 y .88 para cada uno de sus 5 factores, replicando la estructura factorial de 5 factores explicando un 73\% de la varianza (Benet-Martínez \& John, 1998; Rodríguez-Fornells, Lorenzo-Seva \& Andrés-Pueyo, 1999).

\section{Resultados}

\section{Consistencia Interna}

En la estimación de la confiabilidad se realizó a través del Alpha de Cronbach. Para los adultos el alpha obtenido fue de .73, las correlaciones ítem total fluctuaron entre .33 y .67 ; en la muestra de universitarios el alpha obtenido fue de .76 con correlaciones ítem total entre .51 y .66 y para la muestra de adolescentes el alpha fue de .87 con correlaciones ítem total entre .64 y .80 . En la tabla 2 se muestran el detalle de los análisis realizados. 
Tabla 2:

Alpha de Cronbach y Correlación ítem - total para cada una de las muestras estudiadas

\begin{tabular}{|c|c|c|c|}
\hline & Adolescentes & Universitarios & Población General \\
\hline 1. En general, me considero & $.80 * *$ & $.66^{* *}$ & $.67 * *$ \\
\hline 2. En comparación con la mayoría de mis iguales, me considero & $.78 * *$ & $.59 * *$ & $.63^{* *}$ \\
\hline $\begin{array}{l}\text { 3. Algunas personas son muy felices en general. Disfrutan de la vida } \\
\text { independientemente de lo que suceda, sacan el máximo provecho de } \\
\text { todo. ¿Hasta qué punto lo describe esta caracterización? }\end{array}$ & $.67 * *$ & $.54 * *$ & $.50 * *$ \\
\hline \multirow{3}{*}{$\begin{array}{l}\text { 4. Por término general, algunas personas no son muy felices. } \\
\text { Aunque no se encuentran deprimidas, nunca parecen estar tan felices } \\
\text { como podrían. ¿Hasta qué punto lo describe esta caracterización? }\end{array}$} & $.64 * *$ & $.51 * *$ & $.33 * *$ \\
\hline & \multicolumn{3}{|c|}{ Alpha de Cronbach } \\
\hline & Adolescentes & Universitarios & Población General \\
\hline Total & .87 & .76 & .73 \\
\hline
\end{tabular}

$* \mathrm{p}<.05 \quad * * \mathrm{p}<.01$

\section{Validez}

La evaluación de la validez de constructo se realizó por medio del análisis factorial. Los indicadores de adecuación de la matriz de correlación resultaron adecuados para las tres muestras, con índices de KMO entre .70 y .79 y en la prueba de esfericidad de Bartlett todos los casos fueron significativos $(\mathrm{p}<.01)$.
Del análisis factorial por componente principales y rotación varimax, para cada muestra se obtiene una solución de un solo factor que explica entre un $59.92 \%$ y un $72.12 \%$, la saturaciones para este factor fluctúan entre .62 y .90 . El detalle de los resultados obtenidos se muestra en la tabla 3 .

Tabla 3:

Análisis Factorial por componentes principales y Rotación Varimax

\begin{tabular}{|c|c|c|c|}
\hline & Adolescentes & Universitarios & Población General \\
\hline 1. En general, me considero & .90 & .83 & .87 \\
\hline 2. En comparación con la mayoría de mis iguales, me considero & .90 & .79 & .86 \\
\hline $\begin{array}{l}\text { 3. Algunas personas son muy felices en general. Disfrutan de la vida } \\
\text { independientemente de lo que suceda, sacan el máximo provecho de } \\
\text { todo. ¿Hasta qué punto lo describe esta caracterización? }\end{array}$ & .81 & .75 & .76 \\
\hline $\begin{array}{l}\text { 4. Por término general, algunas personas no son muy felices. Aunque } \\
\text { no se encuentran deprimidas, nunca parecen estar tan felices como } \\
\text { podrían. ¿Hasta qué punto lo describe esta caracterización? }\end{array}$ & .79 & .72 & 62 \\
\hline Varianza Total Explicada & $72.12 \%$ & $59.92 \%$ & $61.41 \%$ \\
\hline
\end{tabular}

Los ítems son ordenados en base a cargas factoriales descendentes dominantes $(>0=.40)$.

La evaluación de la validez de criterio se realizó correlacionando la escala de Felicidad con el Cuestionario de Depresión de Beck, las escalas de Neuroticismo y Extraversión del cuestionario de los cinco grandes (BFI) y el cuestionario de Orientación Vital (LOT-R). Todas las correlaciones obtenidas son altamente significativas y la dirección de las correlaciones obtenidas es de acuerdo a lo esperado teóricamente (detalle de las correlaciones obtenidas en Tabla 4). 
Tabla 4:

Correlación entre escala de Felicidad y BDI, neuroticismo, extarversión y LOT

\begin{tabular}{lcccc}
\hline & BDI & Neuroticismo & Extraversión & LOT-R \\
\hline Adultos & $-.485^{* *}$ & $-.247 * *$ & $.274 * *$ & $.364 * *$ \\
Universitarios & $-.399 * *$ & $-.543 * *$ & $.443 * *$ & $.492^{* *}$ \\
Adolescentes & $-.637 * *$ & $-.534 * *$ & $.243 * *$ & $.557 * *$ \\
\hline
\end{tabular}

$* * \mathrm{p}<0.01$

\section{Discusión}

El objetivo de esta investigación fue evaluar las propiedades psicométricas de la escala de felicidad subjetiva, para ello se obtuvieron indicadores en diversas muestras. En un primer estudio se evaluó las propiedades preliminares de la escala (estimación de la confiabilidad y validez de constructo) y un segundo estudio que evaluó las propiedades definitivas de la escala considerando tres grupos etarios (adolescentes, universitarios y población general) de la ciudad de Santiago de Chile. Los resultados obtenidos dan cuenta de adecuadas propiedades psicométricas de esta escala para su aplicación en el contexto nacional.

En detalle se puede señalar que la media en el puntaje de esta escala no se encontraron diferencias significativas entre hombres y mujeres, lo cual coincide con el estudio llevado a cabo por Lyubomirsky \& Lepper (1999). La comparación entre grupo etarios solo se encontraron diferencias significativas entre adultos y adolescentes $(\mathrm{p}<.01)$ y adultos y universitarios $(\mathrm{p}<.01)$ no así entre adolescentes y universitarios $(\mathrm{p}=.42)$, donde la mayor media obtenida por los grupos fue de los adultos $($ Media $=5.33 ;$ D.E. $=1.11)$ y la menor media encontrada fue en el grupo de adolescentes Media $=4.90$; D.E. $=1.12$ )

La evaluación de la consistencia interna fue adecuada tanto en la evaluación preliminar (Alfa $=.78$ ), como en el análisis comparativo entre las muestra de estudio (adultos .73; universitarios .76; adolescentes .87). Respecto a la estabilidad temporal esta fue adecuada con una correlación entre las pruebas de .61 con 8 semanas de diferencias. Similar a indicadores encontrados en otros estudios de estimación de la confiabilidad (Lyubomirsky \& Lepper, 1999).

En cuanto a la validez de constructo, del análisis emerge un solo factor tanto en el análisis preliminar como en la comparación entre los grupos etarios, explicando entre un $59.92 \%$ y un $72.12 \%$, lo cual está en la línea de lo reportado por otros estudios de validación de constructo, donde se presenta una estructura factorial unitaria (Swami, 2008; Swami, et al., 2009)

En cuanto a la validez convergente y divergente la escala de felicidad muestra correlaciones en la línea de lo esperado con las escalas evaluadas. Respecto a la validez convergente se encuentra que las correlaciones alcanzadas con optimismo son moderadas, pero positivas y altamente significativas en los tres grupos etarios estudiados; al igual que con variable extraversión. La validez divergente las correlaciones son de moderadas a buenas, siendo todas negativas y altamente significativas. Estas correlaciones son inferiores a los reportado por Lyubomirsky \& Lepper (1999).

Por tanto, la evidencia indica la adecuación de la escala de felicidad subjetiva para ser usado en la investigación en diversos grupos etarios como una medida confiable y válida para el estudio de felicidad.

\section{Referencias}

Abbe, A., Tkach, C., \& Lyubomirsky, S. (2003). The art of living by dispositionally happy people. Journal of Happiness Studies, 4, 385-404.

Alarcón, R. (2006). Desarrollo de una escala factorial para medir la felicidad. Revista Interamericana de Psicología Interamerican/Journal of Psychology, 40, 99-106.

Alesina, A., Di Tella, R., \& MacCulloch, R. (2004). Inequality and happiness: Are Europeans and Americans different? Journal of Public Economics 88, 2009- 2042.

Barak, Y. (2006). The immune system and happiness. Autoimmunity reviews, 8, 523-7.

Beck, A., Rush, A., Shaw, B., \& Emery, G. (1979). Cognitive therapy of depression. New York:Guildford Press (Spanish Translation in Bilbao: Descleé de Brower, 1983).

Beck, A., Steer, R., \& Garbin, M. (1988). Psychometric properties of the Beck Depression Inventory: Twenty five years of evaluation. Clinical Psychology Review, 8, 77-100.

Benet-Martínez, V., \& John, O. (1998). Los cinco grandes across cultures and ethnic groups: Multitrait multimethod analyses of the big five in Spanish and English. Journal of personality and social psychology, $75,729-750$.

Blanchflower, D. G., \& Oswald, A. (2004). Money, sex and happiness: An empirical study. Scandinavian Journal of Economics 106, 393-415.

Blanchflower, D. G., \& Oswald, A. (2005). Policy forum: Some policy implications of behavioural economics happiness and the human development index: The paradox of Australia. The Australian Economic Review, 38, 307-18.

Buss, D. M. (2000). The evolution of happiness. American Psychologist, $55,15-23$.

Caprara, G.V., Barbaranelli, C., Borgogni, L., \& Perugini, M. (1993). The "Big Five Questionnaire". A new questionnaire to assess the five factor model. Personality and Individual Differences, 15, 281-288.

Chico, E. (2002). Optimismo disposicional como predictor de estrategias de afrontamiento. Psicothema, 14, 544- 550.

Cornelis, J. (2010). Happiness, economics and public policy: A critique review article on Happiness, economics and public policy. En H. Johns 
\& P. Ormerod (edts), The Institute of Economic Affairs, Journal Happiness Study, 1, 125-130.

Cuadra-Peralta, A., Veloso-Besio, C., Ibergaray Pérez, M., \& Rocha Zúñiga, M. (2010). Resultados de la psicoterapia positiva en pacientes con depresión. Terapia Psicologica, 28, 127 - 134.

Csikszentmihalyi, M. \& Hunter, J. (2003). Happiness in everday life: The uses of experience sampling. Journal of Happiness Studies, 4, 1-15

Di Tella, R., MacCulloch, R.J., \& Oswald, A.J. (2003). The macroeconomics of happiness. The Review of Economics and Statistics, 85, 809-827.

Easterlin, R.A. (2001). Life cycle happiness and its sources intersections of psychology, economics, and demography. The economic Journal, 111, 465-484.

Ferrando, P., Chico, E., \& Tous, J. (2002). Propiedades psicométricas del test de optimismo Life Orientation Test. Psicothema, 14,673-680.

Green, C.W., Gardner S. M., \& Reid, D. H. (1997). Increasing indices of happiness among people with profound multiple disabilities: A program replication and component analysis. Journal Of Applied Behavior Analysis 30, 217-228.

Inglehart, R., Foa, R., Peterson, C., \& Welzel, C. (2008). Development, freedom, and rising happiness: A global perspective (1981-2007). Perspectives on a Psychological Science, 3, 264-285.

John, O., \& Srivastava, S. (1999). The big five trait taxonomy: History, measurement and theorical perspectivas. En L. Pervin \& O. John (Edts), Handbook of personality: Theorical and research $\left(2^{\circ}\right.$ ed.). New York: Guilford (In Press).

Johns, H., \& Ormerod, P. (2007). Happiness, economics and public policy. London: The Institute of Economic Affairs.

Joseph, S., Linley, P. A., Harwood, J., Lewis, C.A., \& McCollam, P. (2004). Rapid assessment of well-being: The short depression-happiness scale (SDHS). Psychology and Psychotherapy: Theory, Research and Practice, 77, 463-478.

Kashdan, B.T. (2004). The assessment of subjective well-being (issues raised by the Oxford Happiness Questionnaire). Personality and Individual Differences 36, 1225-1232.

Lewis, C.A., Francis, L.J., \& Zieberts, H.G. (2002). The internal consistency reliability and construct validity of the German translation of the Oxford happiness inventory. North American Journal of psychology 4, 211-220.

Lim, H. (2008). The use of different happiness rating scales: Bias and comparison problem? Social Indicator Research, 87, 259-267.

Lyubomirsky, S. (2008). La ciencia de la Felicidad. Un método probado para conseguir el bienestar. Santiago, Chile: Ediciones Urano.

Lyubomirsky, S., King, L., \& Diener, E. (2005). The benefits of frequent positive affect: Does happiness lead to success? Psychological Bulletin, $131,803-855$

Lyubomirsky, S., \& Lepper, H. S. (1999). A measure of subjective happiness: Preliminary reliability and construct validation. Social Indicators Research, 46, 137-155.

Lyubomirsky, S., Sheldon, K. M., \& Schkade, D. (2005). Pursuing happiness: The architecture of sustainable change. Review of General Psychology, 9, 111-131.

Lyubomirsky, S., \& Tucker, K. L. (1998). Implications of individual differences in subjective happiness for perceiving, interpreting, and thinking about life events. Motivation and Emotion, 22, 155- 186.

Mroczek, D. K., \& Kolarz, C. M. (1998). The effect of age on positive and negative affect: A developmental perspective on happiness. Journal of Personality and Social Psychology, 75, 1333-1349.

Olmedilla-Zafra, A., \& Ortega-Toro, E. (2009). Incidencia de la práctica de actividad física sobre la ansiedad y depresión en mujeres: Perfiles de riesgo. Universitas Psychologica, 8, 105 - 116.

Otake, K., Shimai, S., Tanaka-Matsumi, J., Otsu, K., \& Fredrickson, B.L. (2006). Happy people become happier trough kindess: A counting kindnesses intervencion. Journal of Happines Studies, 7, 361-375.

Otero, J. M., Luengo, A., Romero, E., Gómez, J. A., \& Castro, C. (1998). Psicología de la personalidad. Manual de prácticas. Barcelona: Ariel Practicum.
Pavot, W., \& Diener. E., (1993). The affective and cognitive contest of self reports measures of subjective well-being. Social Indicators Research, 28, 1-20.

Pavot, W. \& Diener. (1999). Review of the satisfaction with life scale. Psychological Assessment, 5, 164-172.

Retana-Franco, B., \& Sánchez-Aragón, R. (2010). Rastreando en el pasado formas de regular la felicidad, la tristeza, el amor, el enojo y el miedo, Universitas Psychologica, 9, 179-197.

Ritterband, L., \& Spielberger, C. (1996). Construct validity of the Beck Depression Inventory as a measure of state and trait depression in nonclinical populations. Depression and Stress, 2, 123-145.

Rodríguez-Fornells, A., Lorenzo-Seva, U., \& Andrés-Pueyo, A. (1999). Psychometric properties of the spanish adaptation of the five factor personality inventory. European of psychological assessment, 7,145-153

Ryan, R. M., \& Deci, E.L. (2001). On happiness and human potentials: A review of research on hedonic and eudaimonic well-being. Annual Review Psychology 52, 141-66.

Ryff, D. (1989). Happiness is everything, or is it? Explorations on the meaning of psychological well-being. Journal of personality and social psychology, 57, 1069-1081.

Sanz, J., \& Vázquez, C. (1998). Fiabilidad, validez y datos normativos del inventario para la depresión de Beck. Psicothema, 10, 303-318.

Scheier, M. F., Carver, C., \& Bridges M. W. (1994). Distinguishing optimism from neuroticism (and trait anxiety, self-mastery, and self-esteem): A reevaluation of the Life Orientation Test. Journal of Personality and Social Psychology, 67, 1063-1078.

Seligman, M. E. P., Steen, T. A., Park, N., \& Peterson, C. (2005). Positive psychology progress empirical validation of interventions. American Psychologist, 60, 410-421.

Steel, P., Schmidt, J., \& Shultz, J. (2008). Refining the relationship between personality and subjective well-being. Psychological Bulletin, $134,138-161$

Swami, V. (2008). Translation and validation of the malay subjective happiness scale. Social Indicator Research, 88,347-353.

Swami, V., Voracek, S. M., Dressler, S. G., Eisma, L., \& Furnham, A. (2009). Psychometric evaluation of the tagalog and german subjective happiness scales and a cross-cultural comparison. Social Indicator Research, 93, 393-406.

Urzúa, A., Cortés, E., Vega, S., Prieto, L., \& Tapia, K. (2009) Propiedades psicométricas del cuestionario de auto reporte de la calidad de vida KIDSCREEN-27 en adolescentes chilenos. Terapia Psicológica, 27, 83-92.

Vázquez, C., \& Sanz, J. (1997). Fiabilidad y valores normativos de la versión española del inventario para la Depresión de Beck de 1978. Clínica y Salud, 8, 403-422.

Vázquez, C., \& Sánz, J. (1999). Fiabilidad y validez de la versión española del inventario para la Depresión de Beck de 1978 en pacientes con trastorno psicológicos. Clínica y Salud, 10, 59-81.

Vera-Villarroel, P., Buela-Casal, G., Zych, I., Córdova-Rubio, N., CelisAtenas, K., Zepeda, L., \& Spielberger, C. D. (2010) Chilean experimental version of the state-trait depression questionnaire (ST-DEP): state subscale (S-DEP). Psychological Reports, 106, 65-77

Vera-Villarroel, P., Córdova-Rubio, N., \& Celis-Atenas, K (2009). Evaluación del optimismo: Un análisis preliminar del Life Orientation Test versión revisada (LOT-R) en población chilena. Universitas Psychologica, 8, 61-68.

Yang, Y. (2008). Social inequalities in happiness in the United States, 1972 to 2004: An age-period-cohort analysis. American Sociological Review, 73, 204-226

Zegers Prado, B., Rojas-Barahona, C., \& Förster Marín, C. (2009). Validez y confiabilidad del índice de satisfacción vital (LSI-A) de neugarten, havighurst \& tobin en una muestra de adultos y adultos mayores en Chile. Terapia Psicológica, 27, 15-26. 